\title{
Testing the accuracy of tropical SST reconstructions from corals
}

\author{
G.Margaritis $^{1}$, E.C. Kent ${ }^{2}$, G.L. Foster ${ }^{1}$, D.I. Berry ${ }^{2}$ \\ ${ }^{1}$ School of Ocean and Earth Science, National \\ Oceanography Centre, University of Southampton, \\ SO143ZH UK \\ ${ }^{2}$ National Oceanography Centre, SO143ZH UK \\ (*correspondence: gm3u13@soton.ac.uk)
}

Records of Sea Surface Temperature (SST) are vital to correctly describe the evolution of the Earth's climate. However, these records suffer from a number of uncertainties including poor metadata and the need to apply bias corrections relating to evolving measurement technique. In light of these uncertainties, geochemical reconstructions of past climate can offer additional, independent, and longer records of SST, as long as their uncertainty is effectively quantified. This study aims to illuminate, quantify and potentially minimize components of uncertainty in SST reconstructions from tropical corals.

Coral-derived SST reconstructions typically rely on older, low-resolution SST products for calibration and validation, without taking into account the discrepancies between gridded SST fields and local, in-situ, sub-surface temperatures as experienced by corals. Furthermore, the reconstruction methodology discards most of the local signal from individual proxies and filters out potentially important information. Our study shows mean monthly differences between high and low resolution SST products and in situ observations can reach up to $\pm 2{ }^{\circ} \mathrm{C}$ and exhibit site-specific and often systematic characteristics. Building on previous studies, we test the sensitivity of geochemical SST reconstructions to the chosen SST product and overall methodology. Subsequently, we first test an approach with an initial, local calibration using new, high-resolution SST products which can potentially preserve important information in the individual proxy records. Secondly, we examine the influence of analytical and chronological uncertainty on the proxy reconstructions. Semi-variogram and Monte Carlo approaches are applied to quantify the most important components of uncertainty in coral-derived SST reconstructions.

Ultimately we aim to identify the optimal method to reconstruct SST using coral-derived SST proxies, including a robust quantification of the uncertainty involved. We will then be able to more robustly extend the observational records and independently test the accuracy of the bias corrections currently applied to historic SST data. 